Maurer School of Law: Indiana University

Digital Repository @ Maurer Law

$9-2020$

\title{
Les Deux Constitutions de John Marshall : une Relecture de l'arrêt Marbury v. Madison
}

Elisabeth Zoller

Maurer School of Law - Indiana University, ezoller@indiana.edu

Follow this and additional works at: https://www.repository.law.indiana.edu/facpub

Part of the Constitutional Law Commons, and the Legal History Commons

\section{Recommended Citation}

Zoller, Elisabeth, "Les Deux Constitutions de John Marshall : une Relecture de l'arrêt Marbury v. Madison" (2020). Articles by Maurer Faculty. 2931.

https://www.repository.law.indiana.edu/facpub/2931

This Article is brought to you for free and open access by the Faculty Scholarship at Digital Repository @ Maurer Law. It has been accepted for inclusion in Articles by Maurer Faculty by an authorized administrator of Digital Repository @ Maurer Law. For more information, please contactrvaughan@indiana.edu. 
ÉLISABETH ZOLLER

De la Cour suprême qu'il présida de 1987 jusqu'à sa mort en 2005, William H. Rehnquist a pu dire :

Il n'est besoin de comprendre que très peu des affaires qu'elle a jugées pour comprendre le rôle de la Cour suprême dans l'histoire de notre nation. Mais l'affaire Marbury contre Madison est une de celles qu'il faut absolument comprendre ${ }^{1}$.

Rien n'est plus juste. Non seulement cette affaire une fois bien comprise apprend beaucoup sur le rôle de la Cour suprême et l'importance du pouvoir judiciaire aux États-Unis, mais aussi, et peut-être plus encore, elle nous instruit beaucoup sur nous-mêmes en nous renvoyant une image inversée de notre propre système juridique.

William Marbury était un homme d'affaires prospère, proche des milieux politiques fédéralistes, qui, lors des élections de 1800, s'était engagé pour la réélection du Président Adams. Celui-ci ne fut pas réélu et, le 4 mars 1801, le républicain Thomas Jefferson lui succéda. Une semaine avant de quitter ses fonctions, Adams désigna Marbury pour occuper les fonctions de juge de paix dans l'une des deux cours que le Congrès venait de créer dans le district de Columbia par une loi du 27 février 1801. Le temps était compté. Le Sénat confirma le candidat désigné, le Président signa l'acte qui affectait Marbury à la cour de district du comté d'Alexandria et l'acte fut transmis dans les tout derniers jours de la présidence Adams au secrétaire d'État, John Marshall, pour être notifié à son bénéficiaire. John Marshall qui attendait de prendre ses nouvelles fonctions (il avait été nommé par Adams et confirmé par le Sénat pour être le prochain président de la Cour

Élisabeth Zoller, Professeur émérite à l'Université Panthéon-Assas (Paris II).

1. W. H. Rehnquist, The Supreme Court, How It Was, How It Is, New York, W. Morrow Co., 1987, p. 99. 
suprême), donna les instructions nécessaires, mais la procédure d'envoi ne fut pas suivie suffisamment de près ${ }^{2}$ et l'acte qui affectait Marbury à la cour d'Alexandria resta en souffrance avec une quarantaine d'autres affectations judiciaires, quand expira le mandat du Président Adams.

Lorsqu'il prit ses fonctions, le Président Jefferson trouva tous ces actes en attente sur son bureau. John Adams avait nommé quarante-deux personnes pour occuper les fonctions de juge de paix dans les comtés de Washington et d'Alexandria du district de Columbia. Toutes avaient été signées et revêtues du sceau officiel, mais elles n'avaient pas été envoyées à leurs destinataires. Jefferson décida de faire envoyer vingt-cinq d'entre elles, mais de retenir les dix-sept autres parce que le nombre de juges lui semblait excessif eu égard aux besoins du district de Columbia. Parmi les juges non retenus, il y avait Marbury et trois autres juges. Ils réclamèrent leurs actes d'affectation en vain, l'administration garda le silence. À la fin de l'année, ils saisirent la Cour suprême d'une demande tendant à ce qu'elle enjoigne à Madison, le nouveau secrétaire d'État, de les leur envoyer. Les treize autres juges ne se joignirent pas à eux, estimant le coût du procès disproportionné pour la modestie de l'office ${ }^{3}$. Là-dessus, en avril 1802, le Congrès annula la session d'été de la Cour suprême qui devait commencer en juin pour la remplacer par une session unique commençant le premier lundi de février de sorte que ce n'est qu'en février 1803 que la Cour put examiner la requête de Marbury.

En principe, la Cour aurait dû immédiatement se déclarer incompétente et rayer l'affaire du rôle. Marbury l'avait saisie en première instance alors qu'elle est une juridiction d'attribution dont les compétences sont définies par la Constitution et qu'elle n'entend en premier ressort que des États ou des cas concernant des ambassadeurs, des ministres publics ou des consuls. Mais la Cour n'alla pas directement au texte; elle fit comme si elle était compétente. Comme l'a écrit Jefferson à la fin de sa vie, «elle voyagea autour de l'affaire ${ }^{4}$ ». Elle demanda à Madison d'expliquer les raisons de son abstention. Madison ne répondit rien, vraisemblablement sur les instructions d'un Président sans illusions quant à l'écoute impartiale qu'il pouvait espérer d'une Cour présidée par un homme qui était l'un de ses «plus farouches ennemis politiques" ». Le

2. Il semblerait que ce soit parce que John Marshall n'attachait pas d'importance aux détails, v. J. A. Garraty, "The Case of Missing Commissions », in Quarrels That Have Shaped the Constitution (J. A. Garraty, ed.), New York, Harper \& Row, 1987, p. 9.

3. A. Beveridge, Life of John Marshall, vol. 3, Boston, New York, Houghton Mifflin Company, 1916, p. 110-111.

4. T. Jefferson, Writings, The Library of America, New York, 1984, Lettre au juge William Johnson, 12 juin 1823 , p. 1474.

5. Un an plus tard, Jefferson a fait part de son amertume à l'épouse du Président Adams, Abigail Adams, qu'il connaissait bien, en ces termes : «En vérité, je peux dire qu'il n’y a qu'un acte, et un seul, dans la vie de M. Adams qui m'ait causé un extrême déplaisir. Je considère que ses dernières nominations furent dirigées contre moi par méchanceté. Il nomma des hommes parmi mes plus farouches ennemis politiques, des hommes dont je ne 
secrétaire d'État ne présenta donc point les arguments républicains. Il y en avait pourtant, de très solides, et ils étaient de notoriété publique.

Les arguments de l'administration républicaine avaient été présentés l'année précédente au Congrès lors de la discussion d'une loi qui rétablissait l'obligation pour les juges de la Cour suprême de siéger dans les cours de circuit et qui supprimait les cours d'appel créées par les Fédéralistes. Ils avaient été exposés par le sénateur du Kentucky, John Breckinridge, porte-parole de Jefferson au Sénat, en ces termes : «La séparation des pouvoirs est conçue pour investir chacun des trois grands départements d'une autorité exclusive sur les matières qui leur sont confiées ${ }^{6}$. » Cette conception était très proche de celle de Bergasse devant l'Assemblée nationale en $1789^{7}$. La séparation des pouvoirs commande l'indépendance réciproque de chaque organe ou branche du gouvernement. Dans le cas d'espèce, elle interdisait à la Cour de se saisir de la demande de Marbury. Jefferson en a donné les raisons de manière limpide : « En matière politique, quel que soit le gouvernement, tout pouvoir indépendant tend à être absolu »; d'où, pour écarter le danger, la nécessité que chaque pouvoir soit totalement indépendant des autres et qu'il ait un droit égal de décider pour lui-même ce que la Constitution lui commande de faire dans les affaires qui ressortent de ses compétences. Et d'expliquer :

Ainsi, dans l'affaire Marbury et Madison, des juges fédéraux prétendaient que des actes de nomination signés par le Président et revêtus de son sceau étaient valables bien que n'ayant pas été envoyés à leurs destinataires. J'ai considéré que l'envoi de l'acte était un élément indispensable pour parfaire l'acte, et que tant qu'il restait entre les mains d'une partie, il n'était pas complet, qu'il était seulement en voie de l'être, mais qu'il ne l'était pas. En conséquence, j'ai suspendu leur envoi. Ils (les juges) ne pouvaient pas prendre d'injonctions contre le Président ou la législature ou contre aucun de leurs agents, la Constitution l'emportant sur la common law dans ces cas précis ${ }^{8}$.

pouvais attendre aucune coopération loyale ", Jefferson T., Writings, op. cit., Lettre à Abigail Adams, 13 juin 1804, p. 1145-1146 (italiques ajoutées).

6. Les propos sont rapportés par A. Beveridge, Life of John Marshall, vol. 3, op. cit., p. 69.

7. «Afin que le pouvoir judiciaire soit organisé de manière à ne mettre en danger ni la liberté civile ni la liberté politique, il faut donc que, dénué de toute espèce d'activité contre le régime politique de l'Etat, et n'ayant aucune influence sur les volontés qui concourent à former ce régime ou à le maintenir, il dispose, pour protéger tous les individus et tous les droits, d'une force telle, que, toute-puissante pour défendre et pour secourir, elle devienne absolument nulle, sitôt que changeant sa destination, on tentera d'en faire usage pour opprimer ", Bergasse, "Rapport sur l'organisation du pouvoir judiciaire », 17 août 1789, Orateurs de la Révolution française (textes présentés par F. Furet et R. Halévi), tome I : Les Constituants, Gallimard, «Bibliothèque de la Pléiade », 1989, p. 107.

8. T. Jefferson, Writings, op. cit., Lettre au juge Spencer Roane, 6 septembre 1819, p. 1426-27 (italiques ajoutées). 
Pour Marbury et son conseil Charles Lee, l'affaire ne soulevait aucune difficulté constitutionnelle. Sa plaidoirie devant la Cour ne faisait aucune allusion à la séparation des pouvoirs. Seule comptait la common law. Le demandeur avait le droit de rejoindre le poste sur lequel il avait été nommé, et il avait utilisé la voie de droit que la procédure de common law offre à un plaideur contre les autorités publiques. Il avait sollicité une injonction, un ordre du juge adressé à une autorité administrative qui la contraint de faire ou de ne pas faire quelque chose. La difficulté - pour autant qu'il y en ait une - ne tenait pas au principe de l'injonction, mais à son application au cas d'espèce. L'injonction était sollicitée contre un membre du cabinet placé directement sous les ordres du Président et la question se posait de savoir si la Cour disposait d'un pouvoir d'injonction contre l'Exécutif, en tant qu'autorité politique traditionnellement protégée par une immunité de juridiction et d'exécution. L'avocat de Marbury pensait que le problème ne se posait pas, la Cour ayant déjà accordé des injonctions pour l'exécution de décisions purement administratives contre des membres du cabinet.

«L'affaire de l'injonction » (mandamus case), comme on l'appelait, était délicate. Qu'allait faire le fédéraliste Marshall dont l'opposition au républicain Jefferson était ancienne et connue 9 ? Allait-il affirmer la compétence de la Cour et prendre le risque que le nouveau Président ignore son arrêt ? Allait-il s'incliner et compromettre l'autorité de la Cour? Les Républicains étaient nerveux à l'idée que la Cour saisisse l'occasion pour donner au pouvoir judiciaire la possibilité de diriger la nouvelle administration dans l'exécution de ses responsabilités officielles ${ }^{10}$. John Marshall se sortit d'affaire par un raisonnement compliqué $^{11}$. Il décida que la Cour n'avait pas de pouvoir d'injonction quand l'exécution relevait de la discrétion de l'Exécutif, lorsqu'il s'agissait de «questions politiques », mais qu'il en allait différemment lorsqu'il s'agissait de questions administratives, par exemple, d'exécuter « un acte administratif » comme en l'espèce où le secrétaire d'État était tenu

9. Sur ce «duel politique et humain», v. R. Le Mestre, «Marbury v. Madison ou Chief Justice Marshall v. President Jefferson? La nécessaire relecture historique et politique d'un arrêt mythique », Rev. Hist. Droit, avr.-juin 2013, vol. 91(2), p. 315. On notera qu'avant d'être nommé Président de la Cour suprême par le Président Adams, John Marshall, avocat de formation, n'avait jamais été juge. Il avait eu une carrière essentiellement politique, tout d'abord en Virginie comme membre du Conseil d'État, ensuite comme représentant élu à l'Assemblée législative de Virginie, et enfin, au niveau fédéral en qualité de diplomate quand il fut nommé par Adams, sur les recommandations de George Washington, envoyé des États-Unis dans la délégation tripartite, dite la mission XYZ, qui devait négocier un compromis commercial avec la France pour éviter la guerre et préserver la neutralité américaine.

10. G. S. Wood, Empire of Liberty, A History of the Early Republic, 1789-1815, Oxford University Press, 2009, p. 403 sq. A titre comparatif, les révolutionnaires français avaient bien pris soin de prévenir un scénario identique avec la loi des 16-24 août 1790 .

11. Marbury v. Madison, 5 U.S (1 Cranch) 137-180 (1803). 
d'envoyer la décision individuelle, la loi l'obligeant à agir dans un sens précis. Dans un deuxième temps, il se demanda si la Cour suprême était bien la juridiction qui pouvait accorder l'injonction sollicitée en application de la loi du Congrès sur le pouvoir judiciaire de 1789. Constatant qu'aux termes de l'article III, sec. 2, de la Constitution, la Cour ne pouvait être saisie en première instance que par des États ou des agents des puissances étrangères, il estima que la loi du Congrès qui lui avait attribué ce pouvoir en première instance était contraire à la Constitution, ce qui posait la question de savoir si une loi contraire à la Constitution pouvait être obligatoire et lier les tribunaux. John Marshall aborda cette question dans un troisième temps et y répondit par la négative, exposant à cette occasion, et pour la première fois de manière particulièrement didactique, la théorie du contrôle judiciaire de constitutionnalité des lois ${ }^{12}$.

Les analyses ou exégèses qui ont été écrites sur ce texte sont innombrables et il n'est pas question ici de prétendre ajouter quelque chose de neuf à une littérature gigantesque. Il s'agit seulement de revenir sur quatre lignes de cette opinion qui ne retiennent pas l'attention alors qu'elles posent une distinction entre deux sortes de constitutions que John Marshall présente comme un préalable à toute sa démonstration de la théorie du contrôle judiciaire de constitutionnalité des lois. Selon lui :

La volonté, originaire et suprême, organise le gouvernement, et assigne leurs compétences respectives aux différents pouvoirs. Elle peut soit s'arrêter là, soit établir des limites que ces pouvoirs ne devront pas dépasser. Le gouvernement des États-Unis ressort de cette dernière catégorie ${ }^{13}$.

À quelles constitutions John Marshall pensait-il ? Un point est certain ; il ne pouvait pas s'agir des constitutions monarchiques comme la constitution d'Angleterre qui n'émanait pas d'une "volonté originaire et suprême ", mais de l'histoire, de la coutume et des usages. Il ne pouvait s'agir que des constitutions républicaines. S'agissant de la seconde, on sait qu'il s'agissait de celle des États-Unis puisqu'il le dit lui-même. Mais quelle était la première? À l'époque, les critères qui

12. Pour une excellente analyse critique des arguments mobilisés par John Marshall pour démontrer l'existence du contrôle judiciaire de constitutionnalité des lois dans la Constitution fédérale des États-Unis, v. I. Fassassi, La légitimité du contrôle juridictionnel de la constitutionnalité des lois aux États-Unis : étude critique de l'argument contre-majoritaire, Dalloz, « Nouvelle bibliothèque des thèses », vol. 165, 2017, p. 74-79.

13. Marbury v. Madison, op. cit., p. 176. 
servent à classer les constitutions aujourd'hui ${ }^{14}$ n'existaient pas. L'existence de "limites à ne pas dépasser » n'était pas ignorée, mais elle était considérée comme incluse dans toute constitution républicaine qui, par hypothèse et par définition, est l'expression d'une « volonté, originaire et suprême », la volonté du peuple, et dont le fonctionnement est sous le contrôle de la volonté populaire par le mécanisme de l'élection, de sorte qu'en principe, en régime républicain, le contrôle de l'excès de pouvoir législatif se fait dans les urnes. Les deux constitutions qu'esquissait John Marshall étaient inhabituelles. Il ne s'agissait certainement pas de «modèles» construits à partir d'un idéal-type comme la doctrine les conçoit de nos jours ${ }^{15}$.

En réalité, ces deux constitutions étaient un habillage de l'opposition entre les deux conceptions de la Constitution fédérale qui s'affrontaient depuis plus de dix ans, celle des Fédéralistes et celle des Républicains. Le conflit était né en 1790 quand Alexandre Hamilton, le secrétaire au Trésor, présenta son rapport sur le crédit public pour résorber la dette de la guerre d'indépendance et qui consistait à s'inspirer de la politique financière suivie en Angleterre au début du XVIII ${ }^{\mathrm{e}}$ siècle. Comme le Premier ministre Robert Walpole, Hamilton proposa de faire racheter les dettes d'États et de particuliers par le gouvernement fédéral et de créer une énorme dette publique qui serait financée par une fiscalité composée d'excises, autrement dit, de droits indirects. Ce programme qui devait faire des États-Unis un État fort, assis sur une fiscalité productive, tournait le dos à l'État modeste, le modèle retenu par Jefferson et Madison pour unir une structure composée d'États qu'ils appelaient une confédération d'États (Confederacy of States) et que, par dérision, Hamilton qualifiait de « gouvernement éloigné et qui ne se voit pas ${ }^{16}$ ». La question politique fondamentale des États-Unis changea de nature ${ }^{17}$. Au

14. Sur les classifications contemporaines des constitutions avec une critique des critères utilisés, v. A. Le Divellec, «De quelques facettes du concept juridique de constitution. Essai de clarification sémantique », Penser le droit à partir de l'individu, Mélanges É. Zoller, Dalloz, 2018, p. 727-753.

15. Sur les modèles, la doctrine française est aujourd'hui plutôt critique alors que la doctrine anglo-américaine y reste attachée. Sur la doctrine française, v. G. Tusseau, Contre les «modèles» de justice constitutionnelle : essai de critique méthodologique - Modelli di giustizia costituzionale : saggion di critica metodologica, édition bilingue français-italien, LGDJ, 2009, et plus récemment les articles de M. Carpentier, "Pour de nouveaux modèles de justice constitutionnelle », Revue internationale de droit comparé, 2016, vol. 68, $\mathrm{n}^{\circ}$ 1, p. 179-219, «Une modélisation alternative des systèmes de justice constitutionnelle : enjeux et difficultés », Revue française de droit constitutionnel, 2019/4, n 120, p. 843-864. Sur la doctrine anglo-américaine, la vogue de la notion de modèle date de S. Gardbaum, "The New Commonwealth Model of Constitutionalism ", American Journal of Comparative Law, 2001, vol. 49, p. 707-760.

16. Lettre $\mathrm{n}^{\circ} 27$ (Hamilton), Le Fédéraliste, traduction Jèze, LGDJ, 1957, p. 213.

17. G. S. Wood, Empire of Liberty, op. cit., p. 151. 
conflit entre Fédéralistes et Anti-fédéralistes se substitua la lutte entre Fédéralistes et Républicains.

Chaque camp concevait la Constitution fédérale comme un acte émanant d'une volonté originaire et suprême. Là où il y avait divergence, c'était sur les limites. Les Fédéralistes plaçaient ces limites dans le pouvoir judiciaire, en particulier, dans le contrôle judiciaire de constitutionnalité des lois ${ }^{18}$. Déjà en 1787, ils avaient beaucoup compté sur les cours de justice pour faire obéir les États et leurs peuples rebelles, peu respectueux des hautes autorités de l'État, trop souvent «turbulents » à leur goût. À l'instar d'Hamilton, ils étaient convaincus que, pour tenir en respect la multitude : "La majesté de l'autorité nationale doit se manifester par le moyen des cours de justice ${ }^{19}$. »

La conception des Républicains était moins aristocratique. Eux aussi voyaient la Constitution comme un acte émanant du peuple, et assorti de limites, mais de limites politiques. Lors du débat précité devant le Congrès, le sénateur Breckinridge avait dit que le contrôle judiciaire de constitutionnalité des lois donnerait aux juges la « direction absolue du gouvernement ${ }^{20}$ » et qu'il le placerait sous « le despotisme d'une oligarchie » comme renchérira Jefferson plus $\operatorname{tard}^{21}$. Pour les Républicains, c'était le suffrage populaire qui limitait les pouvoirs, dans la mesure où des élections régulières obligent les élus à revenir devant les électeurs pour répondre de leurs actes. L'esprit républicain se résumait en un mot d'ordre : « la tyrannie commence là où l'élection annuelle prend fin ${ }^{22}$ ». Jefferson croyait aux vertus du suffrage populaire. À son retour de France en 1790, il avait déclaré aux citoyens du comté d'Albemarle qui le félicitaient pour la manière dont il les avait servis et qui le remerciaient pour son attachement aux droits de l'homme :

Il n'y a que la volonté de la majorité, loi naturelle de chaque société, qui garantisse les droits de l'homme [...] Il convient de s'incliner devant la raison commune de la société parce que, s'il lui arrive de dévier, elle ne tarde pas à revenir dans le droit chemin ${ }^{23}$.

18. Le contrôle judiciaire de constitutionnalité des lois existait déjà dans de nombreux États. Dès 1782, pour endiguer la surenchère des lois, toutes plus démagogiques les unes que les autres, qui étaient adoptées par des assemblées dominées par des factions regroupant toutes sortes d'intérêts particuliers, certains juges avaient dit à leurs législatures élues ce que le juge George Wythe avait dit à la sienne en Virginie : "Là est la limite de vos pouvoirs! Vous pouvez aller jusque-là, mais pas au-delà », v. Commonwealth of Va. v. Caton and Others (Nov. 1782), 4 Call's (Va.) Reports 8 (1782).

19. Lettre $\mathrm{n}^{\circ} 16$ (Hamilton), Le Fédéraliste, op. cit., p. 122.

20. A. Beveridge, Life of John Marshall, vol. 3 (1916), op. cit., p. 69.

21. Lettre de Thomas Jefferson à William Charles Jarvis, 28 septembre 1820, Founders Online, National Archives, https://founders.archives.gov/documents/Jefferson/98-01-021540, site consulté le 29 septembre 2019.

22. Lettre de Thomas Jefferson à Samuel Adams, 26 février 1800, https://founders.archives.gov/documents/Jefferson/01-31-02-0338.

23. T. Jefferson, Writings, op. cit., Response to the Citizens of Albemarle, 12 février 1790, p. 491. 
Les Fédéralistes n'avaient aucune confiance dans des élections régulières, même rapprochées, pour constituer des limites efficaces aux pouvoirs. Pour eux, le peuple était toujours une populace $(\mathrm{mob})$ en puissance. Hamilton était convaincu de « la folie et de la méchanceté des hommes » et de « la perversité de la nature humaine ${ }^{24}$.

La vérité est que les Fédéralistes étaient effrayés des événements que la Révolution française avait déclenchés. Déjà à partir de la révolte des esclaves à Saint-Domingue, le 22 août 1791, et surtout à partir des massacres de septembre 1792, ils associèrent les réformes radicales des révolutionnaires à la Terreur. Le plus grand danger était le jacobinisme, en particulier son athéisme, qu'ils rendaient responsable des désordres et des violences avec ses pernicieuses idées égalitaires ${ }^{25}$. Selon Hamilton, si elle devait se répandre, cette idéologie annonçait pour le monde des maux encore plus grands et compliqués que les trois grands fléaux qui avaient jusqu'alors accablé l'humanité, la guerre, la peste et la famine ${ }^{26}$. Les craintes étaient d'autant plus vives que les idées de la Révolution, que soutenaient Jefferson et les Républicains, rencontraient du succès aux États-Unis parmi les classes populaires qui se sentaient proches des Français dans leur haine de la monarchie. Le livre de Thomas Paine qui avait pris la défense de la Révolution française et des droits de l'homme ${ }^{27}$ contre Edmund Burke et ses Réflexions sur la Révolution ${ }^{28}$ avait soulevé l'enthousiasme. En 1792, Jefferson écrivait à Thomas Paine :

Notre peuple, mon bon ami, est convaincu et unanime dans les principes du républicanisme et la meilleure preuve est qu'ils aiment ce que vous écrivez et vous lisent avec ravissement ${ }^{29}$.

Dans les théâtres, le public debout dans la galerie demandait à l'orchestre sous la menace de la contrainte de jouer le «Ça ira ${ }^{30}$. Le citoyen Genêt, l'agent diplomatique que la Convention avait envoyé aux États-Unis pour rappeler l'alliance entre les deux républiques et obtenir

24. Lettre $\mathrm{n}^{\circ} 78$ (Hamilton), Le Fédéraliste, op. cit., p. 653.

25. G. S. Wood, Empire of Liberty, op. cit., p. 262-263; R. H. Cleves, "Jacobins in this Country" : The United States, Great Britain, and Trans-Atlantic Anti-Jacobinism ", Early American Studies, 2010, vol. 8, p. 433, 436-438 ; J. Den Hartog, "Trans-Atlantic AntiJacobinism : Reaction and Religion ", Early American Studies, 2013, vol. 11, p. 134.

26. A. Hamilton, Writings, New York, The Library of America, 2001, "The "Reynolds Pamphlet" », p. 883

27. T. Paine, Rights of Man (1791), Penguin Classics, 1984.

28. E. Burke, Reflections on the Revolution in France (1790), Penguin Classics, 1968.

29. T. Jefferson, Writings, op. cit., Lettre à Thomas Paine, 19 juin 1792, p. 992.

30. G. S. Wood, Empire of Liberty, op. cit., p. 179. 
le soutien de l'Amérique dans la guerre contre les monarchies d'Europe avait été accueilli par le peuple à bras ouverts. Pis, une société secrète qui encourageait dans tout le pays la formation de clubs sur le modèle de ceux des Jacobins s'était constituée dans une ville comme Philadelphie $^{31}$. Pour empêcher que les États-Unis ne soient entraînés dans un maelstrom identique, les Fédéralistes comptaient deux forces, la religion et le pouvoir judiciaire. En 1797, lorsque la guerre menaçait d'éclater entre la France et les États-Unis, Hamilton voyait dans la première «un moyen important d'influencer l'opinion et une ressource appréciable pour combattre l'athéisme des ennemis ${ }^{32} »$. Quant à la seconde, le pouvoir judiciaire était dans la tradition anglaise le premier moyen pour assurer l'ordre et le gouvernement fédéral ne disposait pas d'autre administration que lui pour faire respecter les lois ${ }^{33}$.

Les Républicains voyaient les choses autrement. Avec Jefferson, ils pensaient qu'un gouvernement républicain, certes, cause de l'agitation parmi le peuple souvent «turbulent », mais que, comparée à l'oppression de ce «gouvernement de loups sur des agneaux » qu'est la monarchie comme disait Jefferson ${ }^{34}$, cette agitation n'était rien, et qu'en réalité, elle était même bénéfique dans la mesure où elle contribuait à maintenir l'intérêt des citoyens pour les affaires de la cité et qu'elle était un signe de vitalité en république. En 1787, Jefferson avait fait part à Madison de sa conviction

qu'une petite rébellion de temps en temps est une bonne chose et qu'elle est aussi nécessaire en politique que les orages le sont dans le monde physique ${ }^{35}$.

La distinction faite par John Marshall entre deux sortes de constitutions n'était donc pas imaginaire ; mais c'était une distinction plus politique que juridique qui correspondait à une opposition d'idées entre deux lectures de la Constitution fédérale. Apparue, on l'a dit, en 1790 à propos de la politique financière d'Hamilton, elle s'était aggravée lorsque

31. A. Beveridge, Life of John Marshall, vol. 2 (1916), op. cit., p. 38.

32. A. Hamilton, Writings, op. cit., Lettre à William Loughton Smith, 10 avril 1797, p. 879 .

33. Les Fédéralistes radicaux qui s'alarmaient de la montée des idées républicaines dans les États pensaient que l'armée pourrait être utilisée pour mater ces aspirations populaires. Mais les plus avisés d'entre eux n'avaient guère d'illusions sur l'irréalisme de ces propositions. Le secrétaire au Trésor, Oliver Wolcott Jr., confia à Fisher Ames en décembre 1799: «Il est impossible dans ce pays de faire de l'armée un bras du gouvernement... Il n'y a pas d'autre moyen de combattre l'opposition des États qu'en mettant en place sur tout le territoire un corps efficace de juges, de magistrats et d'agents fédéraux », G. S. Wood, Empire of Liberty, op. cit., p. 418.

34. T. Jefferson, Writings, op. cit., Lettre à James Madison, 30 janvier 1787, p. 882.

35. T. Jefferson, ibid., p. 882. 
l'opposition entre Fédéralistes et Républicains se radicalisa en 1792 sur la conduite à tenir vis-à-vis de la France. Contre Jefferson qui, nonobstant les excès de la Terreur qu'il déplora, a toujours refusé de condamner la France «parce que la liberté du monde dépendait de l'issue de l'affrontement $^{36}$ ", les Fédéralistes recommandaient une politique de neutralité dans la guerre qui s'engageait en Europe.

L'opposition entre les deux camps avait dominé la présidence Adams et s'était retrouvée au centre des élections de 1800 dont l'enjeu avait porté sur la question de savoir quelle constitution le peuple souhaitait, celle des Fédéralistes ou celle des Républicains. Le verdict des urnes avait été sans appel. Les Républicains avaient gagné de sorte que Jefferson allait pouvoir mettre en œuvre la « révolution de 1800 » qu'il avait annoncée et qui consistait à réduire l'immense influence que les Fédéralistes avaient donnée au pouvoir judiciaire, et à revenir au principe majoritaire, au principe selon lequel, comme l'écrivait Jefferson en 1792 à Gouverneur Morris, le nouvel ambassadeur des États-Unis en France qui lui avait succédé, "la volonté de la nation est la seule chose essentielle à considérer ${ }^{37}$ ». Les Fédéralistes ne l'entendirent point ainsi. Avant de quitter les affaires, ils contrarièrent les projets de Jefferson en fortifiant encore le pouvoir judiciaire, en créant de nouvelles cours, bref, en érigeant, disait la presse favorable au nouveau président, un "Gibraltar » dans les eaux républicaines, d'où ils pourraient tirer sur les réformes de Jefferson ${ }^{38}$.

Pour empêcher que les idées de la Révolution ne gagnent les ÉtatsUnis, les Fédéralistes suivirent les conseils d'Edmund Burke qui avait blâmé les révolutionnaires français d'avoir détruit les Parlements d'Ancien régime. Ils rappelèrent la tradition de common law qui faisait des cours de justice «le plus sûr des asiles contre toutes les révolutions de la mode et de l'opinion ${ }^{39}$ », en particulier, au moyen du contrôle judiciaire de constitutionnalité des lois. Ce pouvoir dit de judicial review était bien connu aux États-Unis ${ }^{40}$. Hérité d'Angleterre où il s'était éteint après la Glorieuse révolution $^{41}$, il était resté vivace outre-Atlantique parce que les

36. T. Jefferson, Writings, op. cit., Lettre à William Short, 3 janvier 1793, p. 1004.

37. T. Jefferson, Writings, op. cit., Lettre à Gouverneur Morris, 30 décembre 1792, p. 1002.

38. J. J. Ellis, American Sphinx, The Character of Thomas Jefferson, Vintage Books, Alfred A. Knopf, 1997 , p. 264.

39. E. Burke, Reflections on the Revolution in France, Penguin Classics, 1968, p. 326 ; Réflexions sur la Révolution de France, traduction française par Pierre Andler, présentation de Philippe Raynaud, Hachette Littératures, « Pluriel », 1989, p. 264.

40. E. S. Corwin, "Marbury v. Madison and the Doctrine of Judicial Review », Michigan Law Review, 1914, vol. 12, p. 538-572.

41. À la fin du XVIII ${ }^{\mathrm{e}}$ siècle, ce contrôle n'existait plus en Angleterre, sinon de manière très allusive, pour faire respecter une règle qui relèverait du droit des gens. Dans un jugement célèbre, Somerset v. Stewart (1772) 98 ER 499, Lord Mansfield avait jugé que l'esclavage était si contraire à toute morale ou politique internationale qu'il ne pourrait être reconnu 
juges d'États avaient vu dans la suprématie de la Constitution le moyen de perpétuer la suprématie du «droit fondamental » (fundamental law) qui protégeait les droits des Anglais (Rights of Englishmen) auxquels ils étaient très attachés ${ }^{42}$. Par ailleurs, l'institution avait servi en 1787 à la Convention de Philadelphie pour garantir la suprématie du droit fédéral sur les lois des États (article VI, sec. 2 de la Constitution).

Mais la Constitution de 1787 ne l'avait pas retenue vis-à-vis des lois du Congrès. Fédéraliste de la première heure, John Marshall semble avoir vu dans l'affaire Marbury l'occasion idoine de combler le vide et de donner, avec toute l'autorité du droit, une autre réponse que la réponse républicaine aux excès de la démocratie. Il entreprit de régler définitivement la controverse entre Fédéralistes et Républicains sur le pouvoir des cours fédérales d'invalider les lois inconstitutionnelles et qui trouve sa source dans ce qu'un ancien attorney general des États-Unis a appelé, il n'y a pas si longtemps, «le problème de la volonté générale ${ }^{43}$ ». C'est que la volonté générale (general will) est toujours un " problème » outreAtlantique. Les Américains restent fidèles à une lecture littérale de Rousseau ${ }^{44}$. À leurs yeux, la volonté générale n'est pas "l'expression de la loi », mais l'expression d'une volonté majoritaire, celle du gouvernement, laquelle, n'étant jamais unanime, n'est qu'une volonté de corps (corporate will) ${ }^{45}$. Pour résoudre le "problème », John Marshall a greffé ou cousu, si l'on préfere, le contrôle judiciaire de constitutionnalité des lois du Congrès sur une Constitution qui n'en parlait pas. Ce fut son chef-d'œuvre. La manière dont il s'y est pris mérite une attention soutenue.

Le conseil de Marbury faisait valoir que la Constitution avait fait de la Cour une "cour suprême 46 ", et qu'à ce titre, comme la cour du banc du roi en Angleterre, " du seul chef de sa suprématie, elle supervise les tribunaux inférieurs et les officiers fédéraux qu'ils soient judiciaires ou

sur la terre d'Angleterre que par une loi expresse du Parlement, ce qui amenait certains juristes à se demander, à supposer qu'elle soit votée, si une telle loi survivrait à un contrôle judiciaire.

42. G. S. Wood, The Creation of the American Republic, 1776-1787, New York, W. W. Norton Co., p. 292.

43. E. H. Levi, « The Sovereignty of the Courts ", University of Chicago Law Review, 1983, vol. 50, p. 679 sq, 683 (italiques ajoutées).

44. R. L. Clinton, "Democracy, the Supreme Court, and Our Two Constitutions", Faulkner Law Review, 2016, vol. 8, p. 1.

45. J.-J. Rousseau, "Du contrat social ", Livre III, chap. 2, Euvres complètes, Du contrat social, Écrits politiques, Gallimard, «Bibliothèque de la Pléiade », 1964, p. 400.

46. Marbury v. Madison, op. cit., p. 146 (en italiques dans le texte). 
administratifs ». S'agissant du pouvoir d'injonction, il soulignait que la Cour avait déjà accordé des injonctions de faire ou de ne pas faire contre des secrétaires du Président et que la demande de son client n'avait rien d'extraordinaire. Le juge Paterson lui demanda s'il considérait que le secrétaire d'État était tenu d'envoyer sa commission à Marbury, à moins que le Président le lui interdise. Lee répondit que lorsque le Président avait signé l'acte de nomination d'un agent sur un poste qu'il ne tenait pas à sa discrétion (comme celui de juge de paix), il avait épuisé ses pouvoirs, que le secrétaire d'État avait compétence liée pour notifier sa nomination à son bénéficiaire et que la loi l'y obligeait. Marbury avait donc, soutenait Lee, un «droit acquis » et, en application de principes toujours suivis par les cours et tribunaux, la Cour devait faire droit à sa demande.

John Marshall s'est bien gardé de disserter sur les conséquences sémantiques que le qualificatif de Cour «suprême » emporte. Il ne s'est pas demandé si la Cour était "suprême » ou non, et si elle avait de ce chef un pouvoir d'injonction contre l'Exécutif. Il s'est demandé si le demandeur avait un droit ${ }^{47}$. Il a renversé les données du problème. Il n'est pas parti du général pour aller au particulier; il a fait l'inverse. Le point de départ de son raisonnement fut la situation concrète de Marbury parce que c'est la démarche naturelle, instinctive, en terre de common law pour aborder un problème juridique, « la première et la plus importante fin du droit étant de maintenir et de réglementer les droits absolus de l'individu ${ }^{48} »$. À cette situation individuelle, il a appliqué les principes invoqués par l'avocat de Marbury, en prenant soin de relever qu'ils avaient été présentés et défendus avec beaucoup de compétence à la barre, mais qu'il allait les présenter dans un ordre un peu différent ${ }^{49}$. Il a fait valoir que

M. Marbury était nommé à partir du moment où son acte d'affectation avait été signé par le Président et que le secrétaire d'État y avait apposé le sceau des États-Unis ; que, la loi qui avait créé cet emploi ayant donné à son bénéficiaire le droit de l'occuper pendant cinq ans sans que l'Exécutif puisse y faire obstacle, la nomination n'était pas révocable, mais qu'elle avait conféré à l'agent des droits acquis protégés par les lois de ce pays. Par conséquent, la rétention de l'acte d'affectation n'était pas autorisée par le droit, mais constituait la violation d'un droit acquis ${ }^{50}$.

47. Ibid., p. 154 .

48. W. Blackstone, Commentaries on the Laws of England, vol. I, book I, chap. 1 (Facsimile of the first edition of 1765-1769), University of Chicago Press, p. 120.

49. Marbury v. Madison, op. cit., p. 154. Quelques années plus tard, John Marshall les qualifia de "principes généraux propres à nos libres institutions ", Fletcher v. Peck, 10 US (6 Cranch), p. 87, 139 (1810).

50. Marbury v. Madison, op. cit., p. 162. 
Autrement dit, Marbury avait un droit, un droit véritable.

Dès lors qu'il avait un droit, John Marshall s'est demandé si

les lois de ce pays offraient à Marbury une voie de recours au cas où ses droits venaient à être violés ${ }^{51}$.

Pour trouver la réponse, il n'est pas allé directement au texte, il n'a pas ouvert la Constitution ; il a utilisé les principes invoqués par l'avocat. Il a convoqué, tout d'abord, le bon sens qui oblige à dire que, s'il n'y avait pas de recours, le pays ne serait pas digne d'être appelé un gouvernement de lois, il ne serait qu'un gouvernement d'hommes; puis, il en a appelé à Blackstone et à ses Commentaires sur les lois d'Angleterre; il a rappelé les usages anglais qui, aussi loin qu'on puisse remonter, ont toujours ratifié le principe qui veut que, là où un droit acquis (vested legal right) est violé, il doit y avoir une voie de droit (remedy) pour le recouvrer, soulignant que lorsqu'il advenait que cette voie de droit ne puisse être donnée par les tribunaux ecclésiastiques, militaires, ou d'amirauté, elle devait être donnée par les cours de common law, selon le principe établi de common law qui veut qu'à chaque droit violé corresponde un recours en justice et que chaque dommage soit redressé par une réparation adéquate; et il a ajouté que même le roi ne manquait jamais de répondre à une pétition ${ }^{52}$.

Et c'est seulement une fois ces principes rappelés que le savant Président s'est, enfin, tourné vers le texte de la Constitution pour savoir si la Cour suprême était bien la juridiction qui pouvait connâtre de la demande de Marbury. Comme on l'a dit, il a répondu par la négative au motif qu'aux termes de l'article III, sec. 2 (2) de la Constitution, la Cour aurait pu l'examiner si la demande lui était venue en appel, mais que lui ayant été adressée en première instance, elle ne pouvait pas en connaître. En termes techniques, John Marshall a distingué entre compétence et recevabilité et, en réalité, il a rejeté la demande pour irrecevabilité, non pour incompétence. En d'autres termes, il a fondé la compétence de la Cour sur la common law.

En fondant la compétence de la Cour sur la common law, autrement dit sur des principes toujours suivis depuis les temps anciens, on oserait à peine dire sur des principes éternels, John Marshall a rappelé que, comme toutes les juridictions de common law, la Cour suprême était l'intermédiaire naturel et obligé entre l'opprimé et le pouvoir oppresseur. L'apport le plus important de Marbury v. Madison est là, dans ce fait très simple d'avoir fondé la compétence de la Cour pour connaître de la requête de Marbury, d'abord, sur la common law et de n'avoir utilisé le 
texte de la Constitution qu'à titre complémentaire, sinon subsidiaire. C'était laisser croire que la compétence de la Cour suprême était de droit commun, non d'attribution. C'était suggérer qu'en tant que cour de common law et comme toutes les cours de common law, la Cour était là pour recevoir les conflits et leur apporter une solution à moins qu'une disposition le lui interdise. Tous les différends, tous les litiges, tous les procès pouvaient virtuellement venir à elle, sauf si la Constitution l'emportait sur la common law dans un cas précis ${ }^{53}$. C'était affirmer que la common law venait avant la Constitution, qu'elle en était le fondement comme elle était le fondement de toutes les lois. À l'instar de celles-ci, la Constitution était posée sur la common law. Son interprétation ou plutôt sa «construction » était prédéterminée par «un corps de lois coutumières, découvertes ou révélées par les cours de justice » et elle devait «trouver sa place dans l'ensemble du droit existant » 54 .

De fait, historiquement, le droit constitutionnel des États-Unis s'est construit sur la common law, ce qui ne signifie pas qu'il ait intégré ses règles de fond. Certes, les Fédéralistes, en particulier, le juge Samuel Chase, a tenté de les imposer à propos des règles sur la diffamation séditieuse. Mais, trois ans plus tard, en 1807, dans le procès d'Aaron Burr, John Marshall a rejeté les règles de la common law anglaise sur la trahison au motif qu'aux termes de l'art. III, sec. 3 (1) de la Constitution, un individu ne peut pas être condamné pour trahison à moins qu'il ait pris les armes contre son pays ${ }^{55}$. Puis, la Cour a exclu les règles de fond de la common law en matière pénale ${ }^{56}$, en matière civile ${ }^{57}$, au motif que les lois fédérales doivent être approuvées par les représentants du peuple (ou ratifiées par leurs représentants, ainsi lorsqu'un État décide de les intégrer au droit d'État).

Toutefois, si le fond de la common law a dû céder devant les exigences de la démocratie, ses formes, ses «structures élémentaires ${ }^{58}$ » relatives

53. Pour Jefferson, c'était le cas de la séparation des pouvoirs, v. supra, note 8 et renvoi au texte correspondant. La précision de Jefferson dans sa lettre au juge Spencer Roane est assez claire : «En conséquence, j'ai suspendu leur envoi. Ils [sous-entendu ici, les juges] ne pouvaient pas prendre d'injonctions contre le Président ou la législature ou contre aucun de leurs agents, la Constitution l'emportant sur la common law dans ces cas précis ", italiques ajoutées.

54. E. Lambert, Le gouvernement des juges et la lutte contre la législation sociale aux ÉtatsUnis. L'expérience américaine du contrôle judiciaire de la constitutionnalité des lois, Paris, Giard, 1921, réédition Dalloz, 2005 (avec une préface de F. Moderne), p. 131.

55. The Aaron Burr Treason Trial (C. F. Hobson, ed.), Federal Judicial Center, Federal Judicial History Office, 2006, https://www.fjc.gov/history/cases/famous-federal-trials/us-vaaron-burr-treason-trial.

56. United States v. Hudson and Goodwin, 11 US (7 Cranch) 32 (1812) qui consacre le principe de légalité des crimes et délits fédéraux.

57. Wheaton v. Peters, 33 US (8 Pet.) 591 (1834) sur la propriété intellectuelle (droits d'auteur).

58. On pense ici bien sûr à C. Lévi-Strauss, Les structures élémentaires de la parenté, Paris, Puf, 1949. 
à la manière dont elle aborde le conflit, pourrait-on dire, habitent le système tout entier. Le "cadre de pensée » (frame of mind) qu'elle impose à l'esprit a survécu ${ }^{59}$. En 1817, John McLean, juge à la Cour suprême de l'Ohio qui rejoindra la Cour suprême en 1830, l'avait compris :

Même si la common law était expressément entièrement abrogée par la loi, seule son ombre disparaîtrait - la vie et l'esprit de la common law survivraient ${ }^{60}$.

Il voyait si juste qu'en 1842, la Cour a ressuscité la vie de la common law en matière commerciale ${ }^{61}$ pour finalement l'abandonner en transférant toute la common law (ou presque) aux États en $1936^{62}$. Mais l'esprit de la common law est resté la marque de l'esprit juridique aux États-Unis. Le résultat est que

celui-ci considère les choses habituellement de manière concrète, non de manière abstraite et qu'il met sa foi plus dans les expériences, que dans les abstractions. C'est un cadre d'esprit qui préfere avancer prudemment sur la base de l'expérience, aller de ce cas-ci ou de ce cas-là au cas suivant, comme la justice l'exige dans chaque cas plutôt d'en revenir toujours à des idées supposées universelles ${ }^{63}$.

En arrimant la Constitution à la common law, John Marshall a fait de la méthode de common law la méthode appropriée pour traiter toute question constitutionnelle, ce qui signifie que celle-ci doit être résolue en partant d'une situation concrète, non d'un énoncé général. Le « cas » du plaignant devenant l'étoile polaire du raisonnement judiciaire, il doit être décidé en le comparant à d'autres - la découverte de similarités ou de différences étant présentée comme "l'étape-clé » de la démarche juridique ${ }^{64}$ - donc en priorité à partir de précédents ${ }^{65}$, et non à partir de textes. Ce fut un coup fatal porté au principe républicain qui tient les textes votés par le peuple souverain pour seule source du droit et qui privilégie la méthode textuelle.

59. R. Pound, "What is the Common Law ? ", University of Chicago Law Review, 1937, vol. 4, p. 181, 186-187. Dans le même sens, on peut dire aussi que la common law est « un état d'esprit ", v. C. Roynier, Le problème de la liberté dans le constitutionnalisme britannique, thèse, Paris-II, 2011, p. 42.

60. W. T. Utter, "Ohio and the English Common Law», Mississippi Valley Historical Review, déc. 1929, vol. 16, p. 321-333, p. 330 (italiques ajoutées). La common law est certainement « un état d'esprit », v. C. Roynier, Le problème de la liberté dans le constitutionnalisme britannique, thèse, Paris-II, 2011, p. 42.

61. Swift v. Tyson, 41 US (16 Pet.) 1 (1842).

62. Erie Railroad Co. v. Tompkins, 304 US 64 (1938).

63. R. Pound, "What is the Common Law?", op. cit., p. 186-187.

64. E. H. Levi, «An introduction to Legal Reasoning ", University of Chicago Law Review, 1948, vol. 15 , p. 502.

65. Dans le même sens, v. D. A. Strauss, «Common Law, Common Ground, and Jefferson's Principles », Yale Law Journal, 2003, vol. 112, p. 1717-1755, p. 1729. 
Une fois que la Constitution fut fondée sur la common law, tout s'en est suivi parce que, en tant que méthode qui façonne un cadre de pensée, la common law enseigne trois choses : 1) la règle du précédent, 2) le jugement par jury, 3 ) la suprématie du droit ${ }^{66}$. Parmi ces trois enseignements, le troisième est décisif pour comprendre le rôle des cours de droit et des juges dans ce système. Il s'est formé en Angleterre au début du $\mathrm{XVII}^{\mathrm{e}}$ siècle à l'occasion de la résistance du grand juge anglais, Sir Edward Coke, contre les prétentions à l'absolutisme des Stuarts ${ }^{67}$. C'est à ce moment-là qu'est née la doctrine selon laquelle le rôle des cours de common law est de s'interposer entre l'individu et toute action oppressive du pouvoir ${ }^{68}$.

Adossée à la common law, la Cour suprême a pu sans difficulté affirmer son pouvoir d'adresser des injonctions au Président car s'il y a bien un domaine où la common law entretient des idées précises, c'est sur le pouvoir du juge de limiter le pouvoir exécutif ${ }^{69}$. De même, elle a pu aussi sans difficulté affirmer le pouvoir du juge de contrôler la constitutionnalité des lois, qui, s'il n'existait pratiquement plus en Angleterre, avait survécu aux États-Unis et avait été déjà reconnu par la Cour suprême elle-même ${ }^{70}$. Dans ces deux domaines, la Cour n'a fait que suivre les traditions de l'histoire et confirmer son autorité comme cour de common law.

À quoi tout ceci nous mène-t-il ? Tout simplement à ce constat qu'en faisant passer la common law avant la Constitution, Marbury v. Madison fut une décision profondément anti-française. Il ne faut ni s'en étonner ni s'en émouvoir. C'était inévitable. Les deux constitutions que John Marshall opposait étaient, d'une part, la «constitution à l'anglaise » ancrée dans l'histoire et la common law et, d'autre part, la «constitution à la française » fondée sur la raison émancipatrice et la volonté d'un peuple libre. Comme l'a si bien expliqué Gordon S. Wood :

66. R. Pound, The Spirit of the Common Law, Marshall Jones Co., Francestown, N. H., 1921, p. 65.

67. V. É. Zoller, Introduction au droit public, Dalloz, 2013, p. 78.

68. R. Pound, The Spirit of the Common Law, op. cit., p. 74.

69. «La common law garantit les droits qui ont été reconnus par les cours de justice. Selon l'interprétation que fait la common law des compétences du pouvoir exécutif, un acte de l'exécutif ne peut jamais retirer des droits de common law, même des droits "ordinaires" (ordinary) [c'est-à-dire, non fondamentaux]. Et la responsabilité de donner effet à certains droits incombent aux cours de justice elles-mêmes à l'occasion des contentieux qui sont portés devant elles », C. Saunders, The Constitution of Australia, A Contextual Analysis, Hart Publishing, Oxford and Portland, Oregon, 2011, p. 268.

70. Hylton v. United States, 3 Dallas 171 (1796). 
La plupart des Fédéralistes étaient convaincus que les principes radicaux de la Révolution française, populaires et égalitaires, menaçaient de corrompre la société américaine et d'en faire une démocratie sauvage et libertine. Ils accusèrent les théories de Voltaire, Rousseau et Condorcet d'avoir infecté la culture des Américains. Les principes de la Révolution française vont nous « détruire en tant que société », dirent-ils, et sont, de ce chef, d'un point de vue moral, autant à redouter que "mille fièvres jaunes pourraient l'être dans la vie physique [...] Mieux vaudrait encore pour les États-Unis périr à jamais que d'être infectés par les principes français ", lançait un Oliver Wolcot hystérique [...]. Ce que les Fédéralistes ne comprenaient pas, c'est que la France n'était nullement la cause des troubles que traversait l'Amérique. Les idées de démocratie et d'égalité qui, à les en croire, affligeaient les États-Unis, n'étaient que la conséquence de leur propre Révolution ${ }^{71}$.

À l'origine, comme beaucoup de ses compatriotes, John Marshall avait accueilli la Révolution avec sympathie. Mais il fut horrifié par la révolte des esclaves à Saint Domingue et le massacre des Blancs égorgés dans leur sommeil "sans considération d'âge ou de sexe », relèvera-t-il plus tard, dans la recension qu'il a faite de la vie de George Washington. À ses yeux, cette tragédie apporta

les fruits précoces et amers d'une philosophie maligne qui, au mépris de l'état actuel du monde, sans la moindre considération pour les misères d'une grande partie de la race humaine, peut froidement et délibérément, au prix de torrents de sang, appliquer des théories abstraites sous prétexte d'atteindre un bien commun jamais expérimenté ;

\section{il fustigea les révolutionnaires français pour avoir}

" conçu le projet fou et maudit de répandre leurs doctrines d'égalité parmi des hommes qui sont séparés par des distinctions et des préjugés qui ne prendront fin que dans la tombe » alors qu'il aurait fallu, selon lui, commencer par corriger les quelques abus qui pouvaient exister, aller à pas lents et prudents, introduire des réformes qui, sans causer la ruine, préparent et adaptent la société aux changements et élargir le cercle du bonheur, sans chercher à atteindre l'impossible ${ }^{72}$.

Il s'est mis à exécrer les idées d'égalité de la Révolution française et à détester Jefferson qui défendait les principes républicains qui en étaient le fondement. Le $1^{\text {er }}$ janvier 1801, alors qu'aucune majorité ne s'était dégagée du collège électoral et que la chambre des Représentants devait

71. G. S. Wood, The Empire of Liberty, op. cit., p. 177-178.

72. J. Marshall, Life of George Washington, Commander in Chief of the American Forces, during the War Which Established the Independence of His Country, and First President of the United States, Philadelphie, 1805 (réédition 1926), vol. IV, p. 459-460. 
départager entre Thomas Jefferson et Aaron Burr pour savoir qui serait le président des États-Unis, il confia dans une lettre à Hamilton :

Vis-à-vis de M. Jefferson dont les idées politiques sont mieux connues que celles de M. Burr, j’ai des objections insurmontables. Ses préjugés en faveur de l'étranger me paraissent le disqualifier pour occuper la magistrature suprême d'une nation qui ne peut pas les adopter sans en subir des conséquences et un dommage permanent ${ }^{73}$.

Hamilton n'en avait pas meilleure opinion qui voyait en Jefferson « un athée en matière religieuse et un fanatique en matière politique » qu'il fallait «empêcher de parvenir à la tête de l'État ${ }^{74}$.

En rappelant l'antériorité séculaire de la common law sur toute constitution écrite, John Marshall a extirpé de la culture juridique américaine les deux plus grandes idées de la Révolution française, la théorie du pouvoir constituant et la théorie de la loi, expression de la volonté générale.

La théorie du pouvoir constituant est rarement acceptée et difficilement compréhensible aux États-Unis non seulement parce que la Constitution est dite et actualisée, en tant que de besoin, par les juges, mais plus encore, parce que la common law maintient à perpétuité l'idée de principes inscrits dans un «droit plus haut ${ }^{75}$ », un droit éternel, que les entreprises humaines ne peuvent pas altérer. Pareille idée est étrangère à la culture juridique française. En 1992, au cours d'une délibération, M. Robert Badinter, alors Président du Conseil constitutionnel, l'a rappelé vertement à un membre de l'institution, M. Jean Cabannes, ancien Premier avocat général à la Cour de cassation, qui défendait l'existence de «principes qui vont au-delà de la Constitution » :

Quoi ? objecta M. Badinter. Il n'y a pas de noyau supraconstitutionnel. C'est un contresens. Dans la bouche d'un homme aussi éminent que vous! Vous le diriez dans un colloque ? C'est inouï d'entendre ça. Où voyez-vous des principes au-delà de la Constitution ?

73. Lettre de John Marshall à Alexandre Hamilton, $1^{\text {er }}$ janvier 1801, Founders Online, National Archives, consulté le 29 septembre 2019, https://founders.archives.gov/documents/Hamilton/01-25-02-0154 [Édition originale : The Papers of Alexander Hamilton, vol. 25, July 1800-April 1802, ed. Harold C. Syrett, New York, Columbia University Press, 1977 , p. 290-292.]

74. A. Hamilton, Writings, op. cit., Lettre à John Jay, 7 mai 1800, p. 924.

75. E. S. Corwin, "The "Higher Law" Background of American Constitutional Law (I et II) », Harvard Law Review, 1928-1929, vol. 42, p. 149-185 et p. 365-409. 


\section{Cabannes avança :}

Nous ne sommes pas dans un colloque, mais il y a bien un droit au-delà du texte de la Constitution [...] il existe des droits essentiels, intangibles.

\section{Le Président Badinter répliqua :}

Mais, bien sûr que non. C'est le constituant qui fixe ce qu'il souhaite. On peut tout réviser, tout. Le souverain c'est le peuple, il est libre, sous réserve de l'article 89 de la Constitution. Le constituant est maître de tout ${ }^{76}$.

Quant à la loi, expression de la volonté générale, elle est tout aussi étrangère à la culture juridique américaine qui n'admet pas que la minorité puisse être liée par la majorité si elle n'est pas d'accord. Mais comment pourrait-il en aller autrement dès lors que la common law ne souscrit pas (et ne peut pas souscrire) à l'idée d'un contrat social unissant les membres de la société 77 , dans la mesure où elle ne raisonne pas à partir des intérêts communs qui unissent les hommes, mais à partir des droits de chacun. L'esprit de common law le commande. En principe, une question de constitutionnalité commence toujours par une affaire individuelle ; elle ne peut venir devant la Cour que sous la forme d'un différend concret qui concerne une situation particulière et la décision qui tranche le cas n'apporte de solution qu'à cette situation ; elle est « un billet de train limité, valable pour un jour et sur ce train seulement ${ }^{78}$ ».

Avec Marbury v. Madison, John Marshall a enrayé la «Révolution de $1800 »$ que Jefferson souhaitait mettre en œuvre s'il était élu. L'inflexion monarchique que les Fédéralistes voulaient imposer aux institutions américaines et que Jefferson, perspicace et visionnaire, avait comprise ${ }^{79}$,

76. CC, décis. no 93-322 DC du 28 juillet 1993, Loi relative aux établissements publics à caractère scientifique, culturel et professionnel, PV 93-07-28, p. 31-32. On rappellera que l'article 89 interdit de réviser la forme républicaine de gouvernement, le principe fondamental auquel Jefferson tenait plus que tout.

77. La majorité ne peut être liée par la minorité que sur le fondement d'un contrat social. La difficulté est qu'il n'y a pas de «contrat social » aux États-Unis. L'idée même fait horreur à certains, notamment les libertariens. Ni les États ni le peuple n'ont mis tout en commun. Les premiers comme le second ont des droits « réservés » (Dixième Amendement). Ils ont certes cherché à rendre l'Union "plus parfaite », mais pas tout à fait.

78. Smith v. Allright, 321 US 649, 669 (1944) (Op. diss. du juge Roberts).

79. T. Jefferson, Writings, op. cit., Lettre à Lafayette, Philadelphie, 16 juin 1792, p. 990 : «Pendant que vous êtes en train d'exterminer la monstrueuse aristocratie et que vous arrachez les crocs et les griffes de la monarchie qui lui est associée, une tendance contraire se fait jour ici. Une secte est apparue parmi nous qui déclare que notre constitution n'est pas assez bonne et qu'elle ne se suffit pas à elle-même, mais qu'elle n'est qu'une étape vers une constitution anglaise, la seule à leurs yeux qui soit bonne et se suffise à elle-même »; Lettre à Thomas Paine, Philadelphie, 19 juin 1792, p. 992 : «Ce n'est que trop vrai que nous avons ici une secte qui prêche les bienfaits d'une constitution à l'anglaise avec un roi, des seigneurs et des communs, et dont les têtes éprouvent des démangeaisons de couronnes, de diadèmes et de mitres. » 
est restée. Les juges sont restés protégés dans la citadelle où les Fédéralistes les avaient installés. Jefferson ne s'y est pas trompé. Vingt ans après que l'affaire eut été jugée, il se plaignait que l'opinion de Marshall dans Marbury v. Madison était sans cesse citée par les avocats comme si elle était un précédent alors qu'elle n'était

qu'un obiter dictum, une dissertation personnelle et hors sujet du Président de la Cour qui n'avait rien à voir avec la question qui était posée ${ }^{80}$.

Il n'avait pas tort, mais tout était déjà joué. L'arrêt était rendu. Le principe selon lequel la Cour est l'interprète ultime et final de la Constitution fut maintes fois invoqué par les parties et confirmé par la Cour à plusieurs reprises si bien que, dès 1833 , Joseph Story, alors juge à la Cour et professeur à Harvard, pouvait écrire "avec confiance » que le principe était acquis ${ }^{81}$. Et, en 1958, par l'effet de la règle du précédent propre à la common law, la Cour suprême pouvait affirmer :

Le principe fondamental que le pouvoir judiciaire fédéral est suprême dans l'interprétation du droit de la Constitution n'a jamais cessé d'être tenu par la Cour et le pays tout entier comme la marque permanente et incontestée de notre système constitutionnel ${ }^{82}$.

À la fin de sa vie, John Adams aurait dit :

Mon don de John Marshall au peuple des États-Unis est l'acte de ma vie dont je suis le plus fier. Il n'y en a pas d'autre auquel je pense avec plus de plaisir. J'ai donné à mon pays un juge égal à un Hole, un Holt ou un Mansfield $^{83}$.

Depuis, les Américains n'en finissent pas de se déchirer sur « l'immense pouvoir judiciaire ${ }^{84}$ » qu'ils ont donné à la Cour suprême et que John Marshall a consolidé et renforcé dans Marbury v. Madison grâce à la common law.

80. Ibid., Lettre au juge William Johnson, 12 juin 1823, p. 1474.

81. J. Story, Commentaries on the Constitution of the United States, Abridged edition by the author, reprinted with an Introduction by R. D. Rotunda et J. E. Nowak, Carolina Academic Press, 1987, p. 132-33.

82. Cooper v. Aaron, 358 US 1, 18 (1958).

83. Ch. Warren, The Supreme Court in United States History, vol. I, op. cit., p. 178.

84. A. de Tocqueville, CEuvres complètes, Gallimard, « Bibliothèque de la Pléiade », tome II, De la démocratie en Amérique, I, I, chap. 8, p. 167. 